\title{
Research Paper: Effectiveness of Neurofeedback Treatment on Adult ADHD: A Meta-Analysis
}

Mohammad Narimani ${ }^{1}$, Elnaz Ensafi ${ }^{*}$, Nastaran Mohajeri Aval

1. Department of Psychology, Faculty of Educational Sciences and Psychology, University of Mohaghegh Ardabili, Ardabil, Iran.

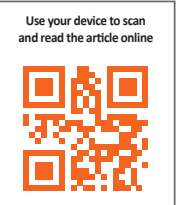

dtation: Narimani, M., Ensafi, E., \& Mohajeri Aval, N. (2018). Effectiveness of Neurofeedback Treatment on Adult ADHD: A Meta-Analysis. Journal of Practice in Clinical Psychology, 6(2), 73-82. https://doi.org/10.29252/nirp.jpcp.6.2.73

Article info:

Received: 13 Sep. 2017

Accepted: 05 Jan. 2018
Keywords:

Meta-analysis, Neurofeedback, Adult, ADHD

\section{ABSTRACT}

Objective: The aim of this meta-analysis was to determine the effectiveness of neurofeedback treatments in adults with Attention Deficit/ Hyperactivity Disorder (ADHD).

Methods: A total of 9 studies were selected, and meta-analysis was done on them. The data were gathered from the following databases: PubMed, ScienceDirect, Springer, SID based on methodological conditions, topic and research design, and the effect size of each study was calculated.

Results: The combined effect size of all the indicators was found to be significant according to Cohen's table, which means large or high effect size. The combined effect size for inattention is $\mathrm{ES}=0.0575(\mathrm{P}=0.0013)$, for impulsivity is $\mathrm{ES}=0.605(\mathrm{P}=0.0037)$, for hyperactivity is $\mathrm{ES}=0.545(\mathrm{P}=0.0384)$, for hyperactivity/impulsivity is $\mathrm{ES}=0.510(\mathrm{P}=0.001)$, and for total $\mathrm{ADHD}$ is $\mathrm{ES}=0.630(\mathrm{P}=0.0038)$.

Conclusion: Based on the results of this meta-analysis, neurofeedback treatment was found to have a large effect in reducing ADHD symptoms in adults with attention deficit/ hyperactivity disorder.

\section{Introduction}

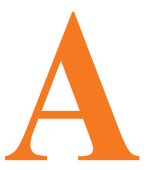

ttention-Deficit/Hyperactivity Disorder (ADHD) is one of the highly prevalent disorders in childhood, and its incidence can be up to $7.5 \%$ by 19 years of age (Barbaresi et al., 2007). The main symptoms of ADHD are inattentiveness, impulsivity, and hyperactivity; these symptoms continue into adulthood in a large proportion of children diagnosed with this disorder (Kessler et al., 2005). The prevalence of adult ADHD in general and clinical populations is estimated to be 4 to $5 \%$ and $7.5 \%$, respectively (Adler, Spencer, Stein, \&
Newcorn, 2008; Goodman \& Thase, 2009). These patients suffer from impairments in educational, occupational, neuropsychological and social functioning (Rostain, 2008). Psychiatric disorders include mood, anxiety and substance abuse are very common in these patients (Sobanski, 2006). ADHD is now considered a chronic disorder that is not limited to childhood only (Barkley, Murphy, \& Fischer, 2010). This change in definition has resulted in the revision of the ADHD diagnostic criteria for older adolescents and adults (i.e. 17 years of age and older) in the recently revised Diagnostic and Statistical Manual of Mental Disorder, fifth edition (Rostain, 2008). Although in many cases, the maladaptive symptoms of

* Corresponding Author:

Elnaz Ensafi, PhD Candidate

Address: Department of Psychology, Faculty of Educational Sciences and Psychology, University of Mohaghegh Ardabili, Ardabil, Iran.

Tel: +98 (45) 33510800

E-mail: elnazensafi89@gmail.com 
impulsivity or hyperactivity are reduced in adulthood, it is incorrectly assumed that the main symptoms of ADHD also disappear (Sobanski, 2006). In addition, the issues faced by adults having ADHD in different situations are often regarded as a part of their individual characteristics and not because of their disorder (Goodman \& Thase, 2009). Therefore, the diagnosis of ADHD is more difficult in adults than in children.

On the functional level, a dysfunction of the prefrontal cortex seems to be the main cause of most of the deficiencies in ADHD, especially the dorsolateral prefrontal cortex and cingulate areas (Makris, Biederman, Monuteaux, \& Seidman, 2009). Electroencephalography (EEG) studies comparing adult individuals with ADHD to healthy controls have suggested a variety of different brain activity patterns including increased theta/beta ratios (Bresnahan, Anderson, \& Barry, 1999; Bresnahan \& Barry, 2002), increased theta and alpha activity (Koehler et al., 2008; White, 2003), and deviant activity in delta and beta frequencies (Clarke et al., 2008). However, the patterns of brain activity seem to be related to the ADHD subtypes (Arns, Conners, \& Kraemer, 2012).

Although medication can improve the major symptoms of ADHD, it cannot completely affect the symptoms and functional deficits (Smith, Mick, \& Faraone, 2009). Moreover, it should be noted that these medications have side effects such as relapse of symptoms when medication stops, drug dependency, reduced appetite, sleeping problem, variation in mood, amenorrhea and palpitation (Barbaresi et al., 2007). Neurofeedback is a treatment method that can be a substitute for pharmacology. Neurofeedback is a self-training treatment that improves brain function by reorganizing the networking and chemical effect of brain. The participants get the ability for changing their brain wave activity by monitoring their brain wave activities in real time via a designed computer device (Swanson et al., 2007). The main assumptions of neurofeedback are consistent with a model that considers ADHD as a disorder involving low brain arousal (Dresler et al., 2010). According to this model, inadequate production or consumption of neurotransmitters causes inefficient delivery among neurons. Neurofeedback allows a participant to enhance connection among neurons through reinforcement and effective transfer of neurons or faster spiking.

Children with ADHD have higher theta wave activity and lower beta wave activity than the normal children (Dresler et al., 2010). A similar pattern can be seen in adults with ADHD (Makris et al., 2009). In other words, the EEG pattern of ADHD is typically characterized by a high rate theta wave activity compared to beta wave activity. Thus, the purpose of neurofeedback training for ADHD is to reduce theta wave activity and increase beta wave activity.

There are limited studies in this area, and we observed that no quantitative meta-analysis has been done on this topic till date. So far, various studies indicate that neurofeedback has been effective in reducing the symptoms of ADHD, but the extent of the impact of these interventions and the role of moderator variables are unclear. Therefore, a meta-analysis serves as a powerful method to integrate many researches and determine their overall effectiveness. This method of analysis allows us to address some of the raised issues and test the effect size -and hence, clinical relevance- of these methods in a quantitative manner. As noted previously, ADHD patients have persistent symptoms of inattention, impulsivity and/or hyperactivity. Thus, in this meta-analysis, we have focused on the effectiveness of neurofeedback treatment on the main symptoms of adult ADHD: hyperactivity, inattention and impulsivity.

In neurofeedback treatment, various therapeutic protocols are used, including SMR enhancement with Theta Suppression, Beta enhancement with Theta suppression, and the training of Slow Cortical Potentials (SCP). In most studies, the central areas $(\mathrm{Cz}, \mathrm{C} 3, \mathrm{C} 4)$ are used as a training site, and only a few studies have used frontal and parietal sites $(\mathrm{Fz}, \mathrm{FCz}, \mathrm{CPz})$. To be in line with the majority of the scientific papers on EEG frequency bands, we classified both SMR/Theta and Beta/Theta training as Beta/Theta training in this meta-analysis because the SMR frequency band $(12-15 \mathrm{~Hz})$ is a part of the Beta-1 frequency spectrum. In addition, several studies have compared theta-beta training and SCP training both within subject 13 and between subjects 12, and both neurofeedback approaches showed comparable effects on the different aspects of ADHD such as inattention, hyperactivity and impulsivity. So, in this meta-analysis, both SCP and theta-beta neurofeedback protocols are investigated in the same analysis.

The following pre- and post-assessment measures were collected from the included studies: 1. Hyperactivity which was assessed with a DSM rating scale such as Conners (CAARS-SS, CAARS-K) or DSM-IV Rating Scale (SCID-I), Mini International Neuropsychiatric Interview (MINI) and the German version of the Wender Utah Adult ADHD structured interview; 2. Inattention which was assessed with an inattention rating scale such as FBB-HKS, Conners (CAARS-K) or DSM-IV Rating Scale (SCID-I) and BAARS-IV; 3. Impulsivity which 
was assessed with Commission errors on a CPT such as a TOVA, IVA (auditory CNV) or Go-No/Go task, and Adult attention deficiency questionnaire; 4. Hyperactivity/Impulsivity which was assessed with ADHD-rating scale self-reported current symptoms for Inattention and Hyperactive/Impulsive (H/I); and 5. ADHD total which was assessed with ADHD-SB.

\section{Methods}

This meta-analysis included all theses, articles and research works available for the period of 1997-2015 on the effectiveness of neurofeedback treatment in adult ADHD. After reviewing the articles, researches, and theses, only 10 research studies (statistical population) with inferential statistics were chosen because they were suitable according to methodological issues and mentioned variable. This statistical population itself was considered as the sample of the study.

The literature was searched for studies investigating neurofeedback or EEG biofeedback in adult ADHD. Furthermore, a search in PubMed, ScienceDirect, Springer, SID, Arak Medical University Journal, and the Journal of Psychology was performed using combinations of the following keywords: 'Neurofeedback' or 'EEG Biofeedback' or 'neurotherapy' or 'SCP' or 'Slow Cortical Potentials' and 'adult ADHD' or 'adult ADD' or 'adult Attention Deficit Hyperactivity Disorder' or 'adult Attention Deficit Disorder'.

All these publications were obtained and screened following the below inclusion criteria: Methodological conditions (as the hypothesis, research methodology, statistical population, sample size, sampling method, the reliability and validity, proper statistical methods); Topic (the effectiveness of neurofeedback treatment in adult ADHD); and The use of experimental or quasi-experimental research design.

Therefore, studies that did not meet the above conditions were omitted from this meta-analysis. In order to choose the appropriate research, the meta-analysis checklist included these titles: researchers, reference, research topic, country, year, medical model, sample size, age, number of neurofeedback sessions, protocols, research tools, and neurofeedback site.

The present meta-analysis followed six steps: 1 . The definition of variables; 2 . Searching databases and sources of information; 3 . Collection of research reports; 4. Calculation of effect size for each study; 5. Combining the effect sizes of studies; and 6. Determination of a sig- nificant amount of combination studies. In the calculation, we did not use certain software and all calculations were done manually.

\section{Results}

Nine studies met all criteria and were included in the meta-analysis. One effectiveness study (Thompson \& Thompson, 1998) and one prospective study (Mayer, Wyckoff, Fallgatter, Ehlis, \& Strehl, 2015) were excluded from the meta-analysis since no SD's were available for those studies. An overview of all included studies can be found in Table 1 . The effect sizes for inattention in 8 studies, impulsivity in 6 studies, hyperactivity in 4 studies, hyperactivity/ impulsivity in 2 studies and self-rated current ADHD symptoms (ADHD-SB) in 2 studies were calculated.

Based on the data presented in each research, the effect size was calculated. Table 2 shows the effect size for each research. Table 3 shows the combined effect size and $\mathrm{p}$-value for inattention, impulsivity, hyperactivity, hyperactivity/impulsivity, and total ADHD. According to Table 3, the combined effect size of all the indicators was found to be significant according to Cohen's table, which means large or high effect size. The combined effect size for inattention is $\mathrm{ES}=0.0575(\mathrm{P}=0.0013)$, for impulsivity is $\mathrm{ES}=0.605(\mathrm{P}=0.0037)$, for hyperactivity is $\mathrm{ES}=0.545(\mathrm{P}=0.0384)$, for hyperactivity/impulsivity is $\mathrm{ES}=0.510(\mathrm{P}=0.001)$, and for total $\mathrm{ADHD}$ is $\mathrm{ES}=0.630$ $(\mathrm{P}=0.0038)$. Therefore, based on the results of this meta-analysis, it can be said that neurofeedback treatment plays a significant role in reducing ADHD symptoms in adults with ADHD.

\section{Discussion}

The statistical method called meta-analysis suggests that the effect sizes are combined to test a particular hypothesis. Thus, a number of research can be done using the same variable, and the results can be combined within a single research (Smith, 2007). The result of this study shows that neurofeedback has a large effect on inattention, impulsivity, hyperactivity, impulsivity/ hyperactivity and ADHD in adults. The results of this meta-analysis are consistent with previous studies (Arns, de Ridder, Strehl, Breteler, \& Coenen, 2009) on the effectiveness of neurofeedback treatment in children with ADHD. Both prospective controlled studies and studies using a pretest and posttest design have found a large effect for neurofeedback on impulsivity and inattention and a medium effect size for hyperactivity. Furthermore, several studies have also reported that the effects of neu- 


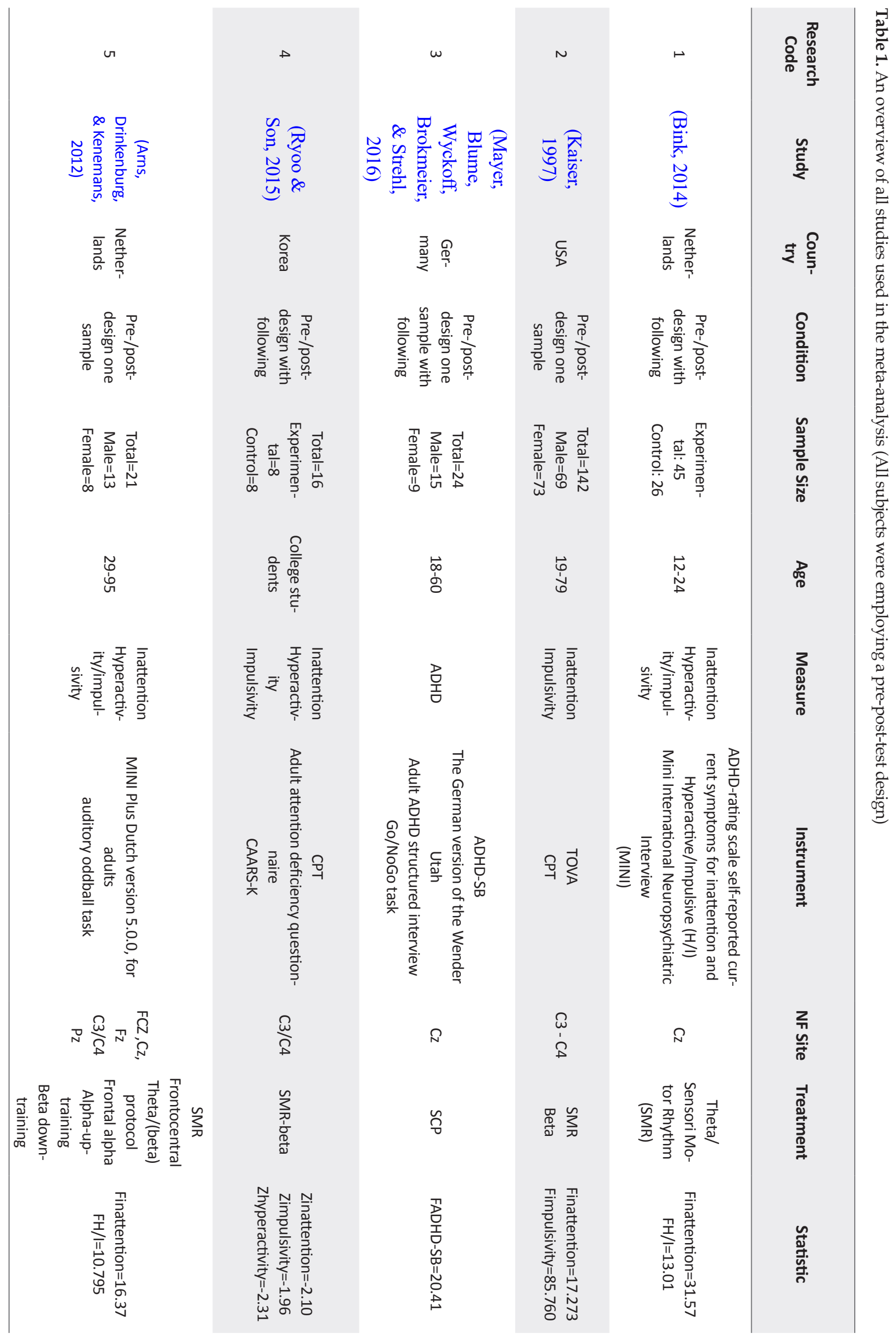




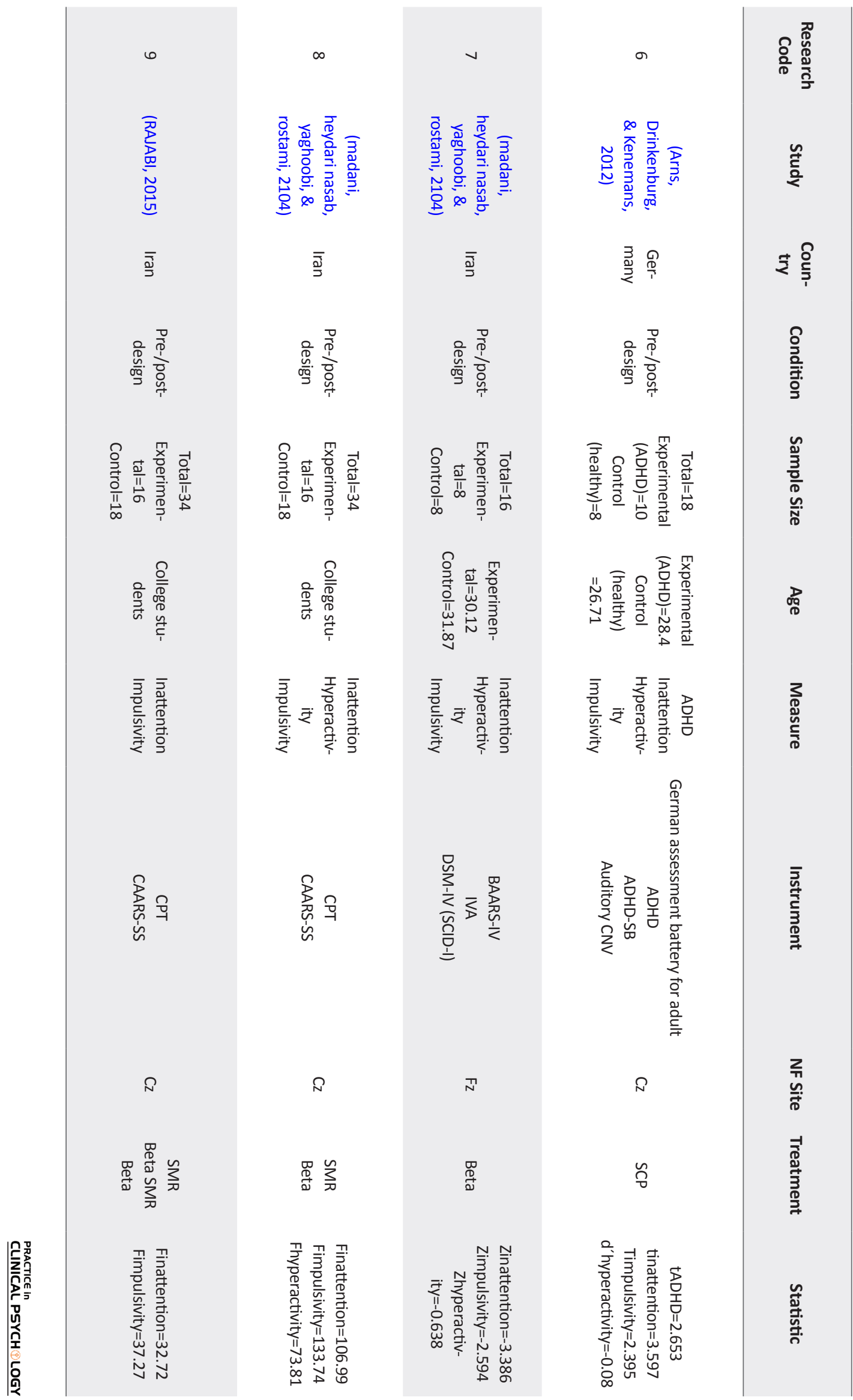


Table 2. The results of meta-analysis of the effectiveness of NF on adult ADHD and the effect size for each research, the conversion of $\mathrm{r}$ to $\mathrm{zr}$ and significance to $\mathrm{z}$

\begin{tabular}{|c|c|c|c|c|}
\hline $\begin{array}{l}\text { Research } \\
\text { Code }\end{array}$ & $\begin{array}{l}\text { Effect Size of NF on Adult ADHD, } \\
\text { Effect Size Based on } r\end{array}$ & The Conversion $r$ to $\mathrm{zr}$ & Sig. & $\begin{array}{l}\text { The Conversion Sig. } \\
\text { to } \mathrm{Z}\end{array}$ \\
\hline 1 & $\begin{array}{l}\text { Rinattention }=0.560 \\
\qquad \mathrm{rH} / \mathrm{l}=0.48\end{array}$ & $\begin{array}{c}\text { Zinattention }=0.633 \\
\text { ZH/I }=0.418\end{array}$ & $P<0.001$ & 3.09 \\
\hline 2 & $\begin{array}{l}\text { Rinattention=0.109 } \\
\text { Rimpulsivity=0.614 }\end{array}$ & $\begin{array}{l}\text { Zinattention }=0.110 \\
\text { Zimpulsivity }=0.717\end{array}$ & $P<0.001$ & 3.09 \\
\hline 3 & $\mathrm{rADHD}=0.607$ & $\mathrm{ZADHD}=0.693$ & $P<0.000$ & 3.70 \\
\hline 4 & $\begin{array}{l}\text { Rinattention=0.245 } \\
\text { Rimpulsivity=0.206 } \\
\text { Rhyperactivity=0.206 }\end{array}$ & $\begin{array}{l}\text { Zinattention=0.250 } \\
\text { Zimpulsivity=0.208 } \\
\text { Zhyperactivity=0.208 }\end{array}$ & $\begin{array}{c}\text { Pinattention=0.035 } \\
\text { Pimpulsivity }=0.012 \\
\text { Phyperactivity=0.012 }\end{array}$ & $\begin{array}{c}\text { Zinattention=1.81 } \\
\text { Zimpulsivity=2.25 } \\
\text { Zhyperactivity=2.25 }\end{array}$ \\
\hline 5 & $\begin{array}{l}\text { Rinattention }=0.690 \\
\qquad \mathrm{rH} / \mathrm{l}=0.612\end{array}$ & $\begin{array}{c}\text { Zinattention }=0.848 \\
\text { ZH/I=0.709 }\end{array}$ & $\begin{array}{l}\text { Pinattention }=0.000 \\
\qquad \mathrm{pH} / \mathrm{l}=0.001\end{array}$ & $\begin{array}{c}\text { Zinattention=3.70 } \\
\mathrm{ZH} / \mathrm{I}=3.09\end{array}$ \\
\hline 6 & $\begin{array}{c}\text { rADHD }=0.662 \\
\text { Rinattention }=0.767 \\
\text { Rimpulsivity }=0.623 \\
\text { Rhyperactivity=-0.039 }\end{array}$ & $\begin{array}{c}\text { ZADHD=0.793 } \\
\text { Zinattention=1.008 } \\
\text { Zimpulsivity=0.725 } \\
\text { Zhyperactivity=-0.039 }\end{array}$ & $\begin{array}{c}\text { PADHD }<0.05 \\
\text { Pinattention }<0.05 \\
\text { Pimpulsivity<0.05 } \\
\text { Phyperactivity }>0.05\end{array}$ & $\begin{array}{c}\text { ZADHD=1.64 } \\
\text { Zinattention=1.64 } \\
\text { Zimpulsivity=1.64 } \\
\text { Zhyperactivity=-1.64 }\end{array}$ \\
\hline 7 & $\begin{array}{l}\text { Rinattention=0.155 } \\
\text { Rimpulsivity=0.200 } \\
\text { Rhyperactivity=0.790 }\end{array}$ & $\begin{array}{l}\text { Zinattention=0.156 } \\
\text { Zimpulsivity=0.203 } \\
\text { Zhyperactivity=1.071 }\end{array}$ & $\begin{array}{c}\text { Pinattention=0.001 } \\
\text { Pimpulsivity=0.010 } \\
\text { Phyperactivity=0.574 }\end{array}$ & $\begin{array}{c}\text { Zinattention=3.09 } \\
\text { Zimpulsivity=2.32 } \\
\text { Zhyperactivity=0.00 }\end{array}$ \\
\hline 8 & $\begin{array}{l}\text { Rinattention=0.876 } \\
\text { Rimpulsivity=0.898 } \\
\text { Rhyperactivity=0.835 }\end{array}$ & $\begin{array}{l}\text { Zinattention=1.354 } \\
\text { Zimpulsivity=1.447 } \\
\text { Zhyperactivity=1.204 }\end{array}$ & $P=0.000$ & $Z=3.70$ \\
\hline 9 & $\begin{array}{l}\text { Rinattention=0.700 } \\
\text { Rimpulsivity=0.723 }\end{array}$ & $\begin{array}{l}\text { Zinattention=0/867 } \\
\text { Zimpulsivity=0.908 }\end{array}$ & $P=0.001$ & $Z=3.09$ \\
\hline
\end{tabular}

PRACTICE In
CLINICAL PSYCH $\oplus$ LOGY

rofeedback training are maintained over a follow-up of 6 months (Gevensleben et al., 2009; Leins et al., 2007). To explain these findings, we can say that NF aims at acquiring self-control over certain brain activity patterns, deriving self-regulation strategies, and implementing these self-regulation skills in daily life. In theta/beta training, children learn to decrease activity in the theta band of the EEG (4-8 Hz) and enhance activity in the beta band $(13-20 \mathrm{~Hz})$.

In the resting EEG, increased slow wave (theta) activity and/or reduced relative alpha $(8-13 \mathrm{~Hz})$ and beta activity were reported in several studies on ADHD children (Banaschewski \& Brandeis, 2007; Barry, Clarke,
\& Johnstone, 2003). Therefore, theta/beta training can target an underlying neuronal dysfunction. In other words, NF can be a means to increase specific cognitive or attentional states (an alert and focused but relaxed state in theta/beta training), regardless of the assumed neurophysiological deviations (Heinrich, Gevensleben, \& Strehl, 2007). SCPs are cortical electrical activity changes that are of several hundred milliseconds to several seconds. They are thought to represent task-dependent short-term mobilizations of cortical processing resources. While negative SCPs demonstrate increased excitation (e.g. during states of behavioral or cognitive preparation), positive SCPs point reduction of cortical excitation of the underlying neural networks (e.g. dur- 
Table 3. The combined effect size and P-value for inattention, impulsivity, hyperactivity. Hyperactivity/ impulsivity and total $\mathrm{ADHD}$, The combines effect size of all the indicators which was significant according to Cohen's table which means large or high effect size

\begin{tabular}{cccc}
\hline Indicator & Number of Study & The Combined Effect Size $(\mathbf{r})$ & The Combined P-Value \\
\hline Inattention & 8 & 0.575 & 0.0013 \\
Impulsivity & 6 & 0.605 & 0.0037 \\
Hyperactivity & 4 & 0.545 & 0.0384 \\
Hyperactivity/ impulsivity & 2 & 0.510 & 0.001 \\
\hline Total ADHD & 2 & 0.630 & 0.0038 \\
\hline
\end{tabular}

ing behavioral inhibition) (Birbaumer, Elbert, Canavan, \& Rockstroh, 1990).

At the neurophysiological level (resting EEG, eventrelated potentials), there is a special relationship between behavioral improvement and theta/beta and SCP training (e.g. association between decrease of theta activity and reduction of ADHD symptomatology) (Gevensleben et al., 2009; Wangler et al., 2011). These neurophysiological effects lead to a better understanding of the underlying mechanisms of the effectiveness of neurofeedback training. On the other hand, a significant improvement in reducing hyperactivity symptoms during the neurofeedback training may represent significant attentional processes in reducing hyperactivity symptoms. This is because the self-regulatory mechanisms of brain waves that play a fundamental role in normal function of the brain improve attention and decrease hyperactivity in students (Demos, 2005).

The human brain is capable of healing on their own and has the ability to learn or relearn self-regulatory brain wave mechanisms that have an important role in normal brain functioning (Demos, 2005). So, neurofeedback training strengthens the underlying mechanisms of selfregulation to work effectively. This education system with feedback to the brain about what the person has done in the last few seconds and what the bioelectrical rhythm of the normal brain were encourages the brain to correct, modify and maintain appropriate activity.

The theoretical basis of neurofeedback is related to the law of effect and learning theories such that rewarding a particular behavior will increase the likelihood of recurrence behavior (Thompson \& Thompson, 2003). Operating conditioning process includes behavior modification by which the consequences of an action specify the possibility of the behavior in the future. The abundance of positively reinforced behaviors increases in the future while behaviors that are negatively reinforced will disappear (Gazzaniga, Heatherton, \& Veronese, 2003). It seems that the principles of factor conditioning are an important factor in the ability of neurofeedback to make changes in EEG. So, neurofeedback provides continuous information for the person after expressing the desired behavior so that this information will lead to a recurrence of that behavior.

The results of this meta-analysis support the value and effectiveness of neurofeedback treatment in adults with ADHD. It can be concluded that neurofeedback treatment can help people with ADHD in regulating the activity of brain waves and, thus, improve their impulsivity, inattention and hyperactivity problems.

One limitation of this study is that it is difficult to perform a comprehensive and accurate comparison among all the treatment approaches in adult ADHD due to the limited number of studies on neurofeedback treatment in adult ADHD. Also, due to the limited number of studies, comparisons between integrated approaches with non-integrated approaches have not been done. In this meta-analysis, the researchers tried to use all researches done at a specified time interval. However, there are definitely some other studies that are beyond the scope of this study. Also, different researches will be done in the coming years in the field of the effectiveness of neurofeedback on adult ADHD. On the other hand, due to the lack of access to all previous researches, it was impossible to control interventional variables, such as the age of subjects. Hence, it is suggested that future research should control the intervening variables by visiting universities and reviewing dissertations. The most important limitation of this study was the lack of access 
to research resources for researchers and lack of some statistical findings in journals. Failure to report accurate indications related to hypothesis testing in many researches was led to their exclusion. It is recommended that researchers should completely mention the results of the analysis of their assumptions and with a significant level and not just the overall results. Researchers should also calculate effect sizes and note them in their findings if they find a relationship between variables. Therefore, it is suggested that future meta-analyzes should gather information from more extensive sources.

In conclusion, we provide evidence for the effectiveness of neurofeedback treatment on adult ADHD. By statistical explanation, the combined effect size of all the indicators (inattention, impulsivity, hyperactivity, hyperactivity/impulsivity, total ADHD) was found to be significant according to Cohen's table, which is similar to the values predicted by retrospective studies.

\section{Acknowledgments}

We hereby would like to acknowledge Mohaghegh Ardabili University and all authors who helped us with this meta-analysis project and provided us with some useful feedback and comments in this regard.

\section{Conflict of Interest}

The authors declared no conflicts of interest.

\section{References}

Adler, L. A., Spencer, T., Stein, M. A., \& Newcorn, J. H. (2008). Best practices in adult ADHD: Epidemiology, impairments and differential diagnosis. CNS Spectrums, 13(10 Suppl 15), 4. doi: $10.1017 /$ s1092852900003217

Arns, M., Conners, C. K., \& Kraemer, H. C. (2012). A decade of EEG Theta/Beta ratio research in ADHD. Journal of Attention Disorders, 17(5), 374-83. doi: 10.1177/1087054712460087

Arns, M., De Ridder, S., Strehl, U., Breteler, M., \& Coenen, A. (2009). Efficacy of neurofeedback treatment in ADHD: The effects on inattention, impulsivity and hyperactivity: A meta-analysis. Clinical EEG and Neuroscience, 40(3), 180-9. doi: $10.1177 / 155005940904000311$

Arns, M., Drinkenburg, W., \& Leon Kenemans, J. (2012). The effects of QEEG-informed neurofeedback in ADHD: An openlabel pilot study. Applied Psychophysiology and Biofeedback, 37(3), 171-80. doi: 10.1007/s10484-012-9191-4

Banaschewski, T., \& Brandeis, D. (2007). Annotation: What electrical brain activity tells us about brain function that other techniques cannot tell us? A child psychiatric perspective.
Journal of Child Psychology and Psychiatry, 48(5), 415-35. doi: 10.1111/j.1469-7610.2006.01681.x

Barbaresi, W., Katusic, S., Colligan, R., Weaver, A., Pankratz, V., Mrazek, D., et al. (2007). How common is attention-deficit/ hyperactivity disorder? Towards resolution of the controversy: results from a population-based study. Acta Paediatrica, 93, 55-9. doi: 10.1111/j.1651-2227.2004.tb03058.x

Barkley, R. A., Murphy, K. R., \& Fischer, M. (2010). ADHD in adults: What the science says. New York: Guilford Press.

Barry, R. J., Clarke, A. R., \& Johnstone, S. J. (2003). A review of electrophysiology in attention-deficit/hyperactivity disorder: I. Qualitative and quantitative electroencephalography. Clinical Neurophysiology, 114(2), 171-83. doi: 10.1016/s13882457(02)00362-0

Bink, M. (2014). Shifting attention to neurofeedback: Psychophysiology in adolescents with ADHD and autism spectrum disorders (PhD Thesis). Tilburg: Tilburg University.

Birbaumer, N., Elbert, T., Canavan, A. G., \& Rockstroh, B. (1990). Slow potentials of the cerebral cortex and behavior. Physiological Reviews, 70(1), 1-41. doi: 10.1152/ physrev.1990.70.1.1

Bresnahan, S. M., \& Barry, R. J. (2002). Specificity of quantitative EEG analysis in adults with attention deficit hyperactivity disorder. Psychiatry Research, 112(2), 133-44. doi: 10.1016/ s0165-1781(02)00190-7

Bresnahan, S. M., Anderson, J. W., \& Barry, R. J. (1999). Agerelated changes in quantitative EEG in attention- deficit/hyperactivity disorder. Biological Psychiatry, 46(12), 1690-7. doi: 10.1016/s0006-3223(99)00042-6

Clarke, A. R., Barry, R. J., Heaven, P. C., McCarthy, R., Selikowitz, M., \& Byrne, M. K. (2008). EEG in adults with attentiondeficit/hyperactivity disorder. International Journal of Psychophysiology, 70(3), 176-83.

Demos, J. N. (2005). Getting started with neurofeedback. New York WW Norton \& Company.

Dresler, T., Ehlis, A. C., Heinzel, S., Renner, T. J., Reif, A., Baehne, C. G., et al. (2010). Dopamine transporter (SLC6A3) genotype impacts neurophysiological correlates of cognitive response control in an adult sample of patients with ADHD. Neuropsychopharmacology, 35(11), 2193-202. doi: 10.1038/npp.2010.91

Gazzaniga, M. S., Heatherton, T. F., \& Veronese, M. A. V. (2003). Psychological science: Mind, Brain, and Behavior. New York: WW Norton \& Company.

Gevensleben, H., Holl, B., Albrecht, B., Vogel, C., Schlamp, D., Kratz, O., et al. (2009). Is neurofeedback an efficacious treatment for ADHD? A randomised controlled clinical trial. Journal of Child Psychology and Psychiatry, 50(7), 780-9. doi: 10.1111/j.1469-7610.2008.02033.x

Goodman, D. W., \& Thase, M. E. (2009). Recognizing ADHD in adults with comorbid mood disorders: Implications for identification and management. Postgraduate Medicine, 121(5), 31-41. doi: 10.3810/pgm.2009.09.2049

Heinrich, H., Gevensleben, H., \& Strehl, U. (2007). Annotation: Neurofeedback? Train your brain to train behaviour. Journal of Child Psychology and Psychiatry, 48(1), 3-16. doi: 10.1111/j.1469-7610.2006.01665.x 
Kaiser, D. A. (1997). Efficacy of neurofeedback on adults with attentional deficit and related disorders. Granada Hills: EEG Spectrum Inc.

Kessler, R. C., Adler, L. A., Barkley, R., Biederman, J., Conners, C. K., Faraone, S. V., et al. (2005). Patterns and predictors of attention-deficit/hyperactivity disorder persistence into adulthood: Results from the national comorbidity survey replication. Biological Psychiatry, 57(11), 1442-51. doi: 10.1016/j. biopsych.2005.04.001

Koehler, S., Lauer, P., Schreppel, T., Jacob, C., Heine, M., Boreatti-Hümmer, A., et al. (2008). Increased EEG power density in alpha and theta bands in adult ADHD patients. Journal of Neural Transmission, 116(1), 97-104. doi: 10.1007/s00702-0080157-x

Leins, U., Goth, G., Hinterberger, T., Klinger, C., Rumpf, N., \& Strehl, U. (2007). Neurofeedback for children with ADHD: A comparison of SCP and Theta/Beta protocols. Applied Psychophysiology and Biofeedback, 32(2), 73-88. doi: 10.1007/s10484007-9031-0

Madani, A., Heydari Nasab, L., Yaghoobi, H., \& Rostami, R. (2104). [Evaluate the effectiveness of neurofeedback on reducing attention and concentration deficit symptoms and reducing impulsivity and hyperactivity symptoms in adult ADHD (Persian)]. Scientific-Research Journal of Shahed University, 21(11), 85-98.

Makris, N., Biederman, J., Monuteaux, M. C., \& Seidman, L. J. (2009). Towards conceptualizing a neural systems-based anatomy of attention-deficit/hyperactivity disorder. Developmental Neuroscience, 31(1-2), 36-49. doi: 10.1159/000207492

Mayer, K., Blume, F., Wyckoff, S. N., Brokmeier, L. L., \& Strehl, U. (2016). Neurofeedback of slow cortical potentials as a treatment for adults with attention deficit-/hyperactivity disorder. Clinical Neurophysiology, 127(2), 1374-86. doi: 10.1016/j. clinph.2015.11.013

Mayer, K., Wyckoff, S. N., Fallgatter, A. J., Ehlis, A. C., \& Strehl, U. (2015). Neurofeedback as a nonpharmacological treatment for adults with Attention-Deficit/Hyperactivity Disorder (ADHD): Study protocol for a randomized controlled trial. Trials, 16(1). doi: 10.1186/s13063-015-0683-4

Mayer, K., Wyckoff, S. N., Schulz, U., \& Strehl, U. (2012). Neurofeedback for adult attention-deficit/hyperactivity disorder: Investigation of slow cortical potential neurofeedbackpreliminary results. Journal of Neurotherapy, 16(1), 37-45. doi: 10.1080/10874208.2012.650113

Narimani, M., Rajabi, S., \& Delavar, S. S. (2013). Effects of neurofeedback training on female students with attention deficit and hyperactivity disorder. Arak Medical University Journal, 16(2), 91-103.

RAJABI, S. (2015). Efficacy of neurofeedback in the correct brain's wave's amplitude and the visual-motor continuous performance with ADHD. Journal of Psychology, 19(1): 53-70.

Rostain, A. L. (2008). Attention-deficit/hyperactivity disorder in adults: Evidence-based recommendations for management. Postgraduate Medicine, 120(3), 27-38. doi: 10.3810/ pgm.2008.09.1905

Ryoo, M., \& Son, C. (2015). Effects of neurofeekback training on EEG, Continuous Performance Task (CPT), and ADHD symptoms in ADHD-prone college students. Journal of Korean Academy of Nursing, 45(6), 928. doi: 10.4040/jkan.2015.45.6.928
Seipp, B. (1991). Anxiety and academic performance: A meta-analysis of findings. Anxiety Research, 4(1), 27-41. doi: $10.1080 / 08917779108248762$

Smith, A. K., Mick, E., \& Faraone, S. V. (2009). Advances in genetic studies of attention-deficit/hyperactivity disorder. Current Psychiatry Reports, 11(2), 143-8. doi: 10.1007/s11920-0090022-0

Smith, J. A. (2007). Qualitative psychology: A practical guide to research methods. Thousand Oaks, California: Sage.

Sobanski, E. (2006). Psychiatric comorbidity in adults with Attention-Deficit/Hyperactivity Disorder (ADHD). European Archives of Psychiatry and Clinical Neuroscience, 256(S1), i26-31. doi: 10.1007/s00406-006-1004-4

Swanson, J. M., Kinsbourne, M., Nigg, J., Lanphear, B., Stefanatos, G. A., Volkow, N., et al. (2007). Etiologic subtypes of attention-deficit/hyperactivity disorder: Brain imaging, molecular genetic and environmental factors and the dopamine hypothesis. Neuropsychology Review, 17(1), 39-59. doi: 10.1007/s11065-007-9019-9

Thompson, L., \& Thompson, M. (1998). Neurofeedback combined with training in metacognitive strategies: Effectiveness in students with ADD. Applied Psychophysiology and Biofeedback, 23(4), 243-63. doi: 10.1023/a:1022213731956

Thompson, M., \& Thompson, L. (2003). Neurofeedback treatment for autistic spectrum disorders: Review of 60 cases-principles and outcome. Applied Psychophysiology and Biofeedback, 28(4), 305-6.

Wangler, S., Gevensleben, H., Albrecht, B., Studer, P., Rothenberger, A., Moll, G. H., \& Heinrich, H. (2011). Neurofeedback in children with ADHD: Specific event-related potential findings of a randomized controlled trial. Clinical Neurophysiology, 122(5), 942-50. doi: 10.1016/j.clinph.2010.06.036

White, J. N. (2003). Comparison of QEEG reference databases in basic signal analysis and in the evaluation of adult ADHD. Journal of Neurotherapy, 7(3-4), 123-69. doi: 10.1300/ j184v07n03_06 
